\title{
Research Paper: \\ Evaluation of Cytotoxicity and Molecular Docking Studies of Phthalimide and Naphthalimide Derivatives as Potential Anticancer Agents
}

\author{
Negin Ahanj ${ }^{1}$, Mehdi Taghavi² ${ }^{2}$, , Ayyub Mojaddami ${ }^{3}$ (1)
}

1. Toxicology Research Center, Medical Basic Sciences Research Institute, Ahvaz Jundishapur University of Medical Sciences, Ahvaz, Iran. 2. Department of Chemistry, Faculty of Sciences, Shahid Chamran University of Ahvaz, Ahvaz, Iran.

3. Department of Medicinal Chemistry, School of Pharmacy, Ahvaz Jundishapur University of Medical Sciences, Ahvaz, Iran.

\begin{tabular}{l|l|l|l|l}
\hline $\begin{array}{l}\text { Use your device to scan } \\
\text { and read the article online }\end{array}$ & $\begin{array}{l}\text { Citation Ahanj N, Taghavi M, Mojaddami A. Evaluation of Cytotoxicity and Molecular Docking Studies of Phthalimide and Naph- } \\
\text { thalimide Derivatives as Potential Anticancer Agents. Jundishapur Journal of Medical Sciences. 2021; 20(4):366-375. https://doi. } \\
\text { org/10.32598/JSMJ.20.4.2456 }\end{array}$ \\
dol https://doi.org/10.32598/JSMJ.20.4.2456
\end{tabular}

Received: 22 Apr 2021 Accepted: 21 Aug 2021 Available Online: 01 Oct 2021

Keywords:

Anti-cancer, Naphthalimide derivatives, Phthalimide derivatives, Molecular docking, MTT test

\section{A B STRACT}

Background and Objectives Cancer is the second leading cause of death in the world after cardiovascular disease, so the aim of the present study was to investigate phthalimide and naphthalimide derivatives in order to develop anticancer compounds.

Subjects and Methods In this study, the cytotoxic activity of six phthalimed and naphthalamide derivatives was evaluated using MTT method on three cancerous cell lines, including breast cancer (MCF-7), ovarian cancer (SKOV3) and lung cancer (A549) cell line. Molecular Docking studies were also performed to determine the binding energy and the compounds interaction with DNA as a possible target of these compounds.

Results Based on MTT results, compound C1, a naphthalimide derivative, showed the highest cytotoxic activity. IC50 values of this compound against MCF-7, SKOV3 and A549 cancer cell lines were 1.7, 6.2 and $9.5 \mu \mathrm{M}$, respectively. Also, comparison of phthalimide and naphthalimide derivatives showed that compounds C1, C3, C5 with carboxyl group had better effects than other compounds, C2, C6, C4, which bearing 5-amidoisophthalic acid moiety.

Conclusion In general, naphthalimide derivatives showed better cytotoxicity than phthalimide derivatives. Compound $\mathrm{C} 1$ has the highest cytotoxic activity on all three cancer cell lines and can be further studied in the development of new anti-cancer compounds.

\section{* Corresponding Author:}

Address: Department of Medicinal Chemistry, School of Pharmacy, Ahvaz Jundishapur University of Medical Sciences, Ahvaz, Iran. Tel: +98 (917) 0457581

E-Mail: mojaddami.a@gmail.com 


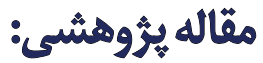 \\ بررسى سميت سلولى و مطالعات داكينگ مولكولى بر روى مشتقات فتاليميد و نفتاليميد به عنوان

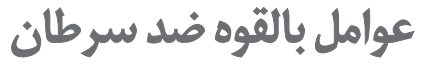 نكين آهنج'، مهدى تقوى ']ـ، "ايوب مجدمى"

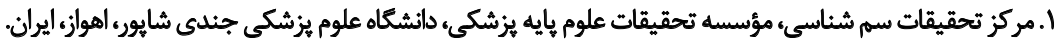

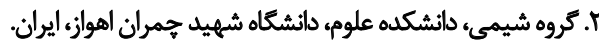

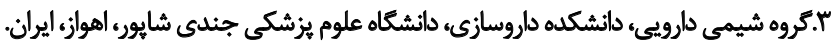

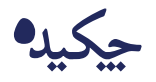

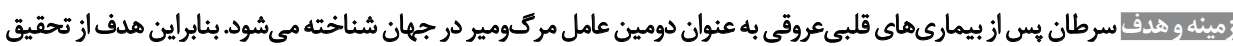
حاضر، بررسى مشتقات فتاليمد و نفتاليميد به منظور ثوسعه تركيبات ضدوند سرطان است.

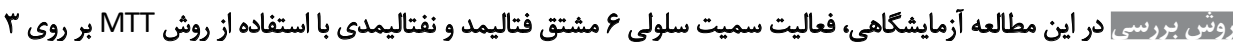

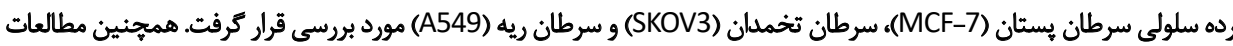

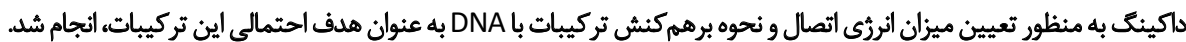

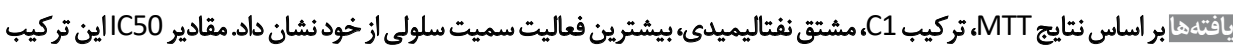

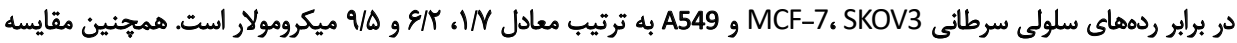

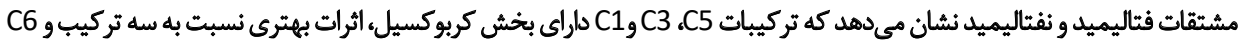

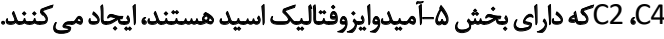

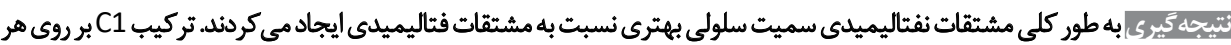

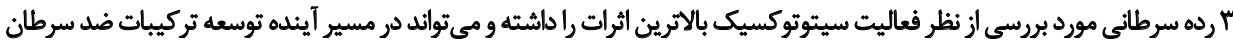

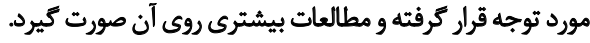

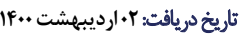

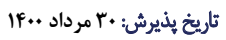

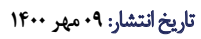

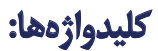

ضد سرطان مشتقات نفتاليميدى، مشتئقات فتاليميدى، داكينك مولكولى، تست فاكيني

سنتزى يافت مىشوند كه اين هستهها فعاليتهاى بيولوزيكى

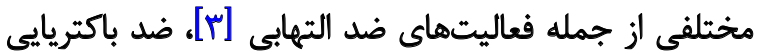

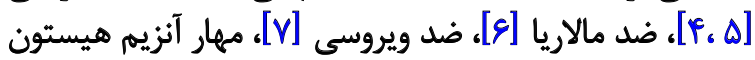

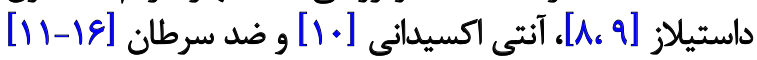
به اين مشتقات مى بخشد.

داروهاى ضد سرطان اهداف مختلفى دارند كه در اين ميان،

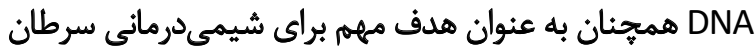

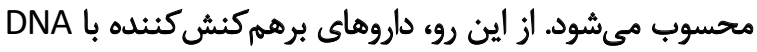

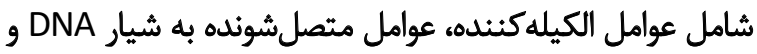

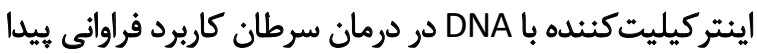

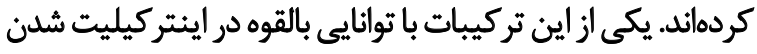

سرطان يك مسئله عمله بهداشت عمومى در سراسر جهان

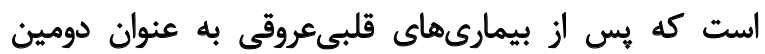

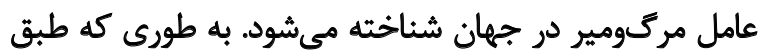

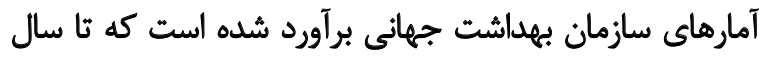

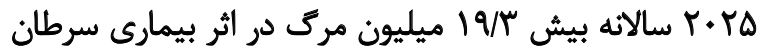

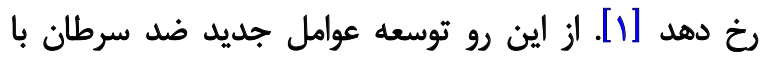

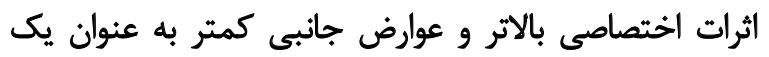

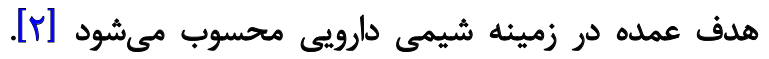

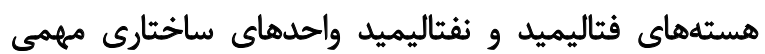

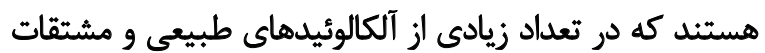

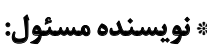

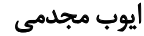

نشائي:كروه شيمي دارويي، دانشكده داروسازي، دانشكاه علوم برُشكي جندي شايور، اهوازئ ايران

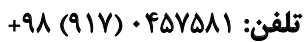

رايانامه: mojaddami.a@gmail.com 
بروسى فعاليث سيثوثوكسيك به روش MTT در اين مطالعه تجربى سميت سلولى ع تركيب فتاليميدى و

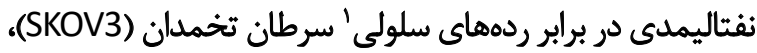

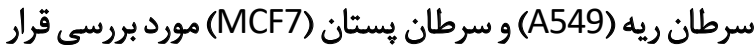

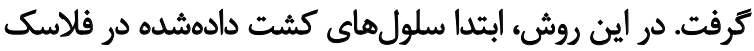

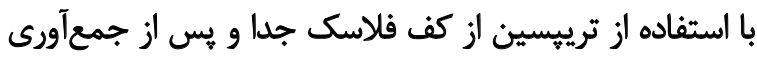

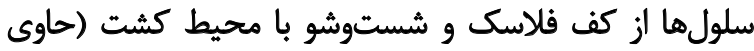

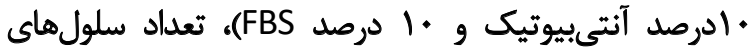

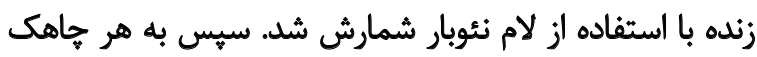

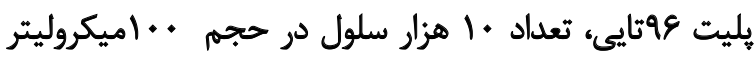

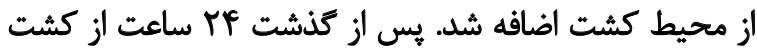

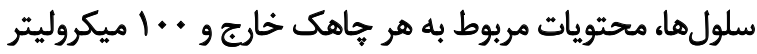

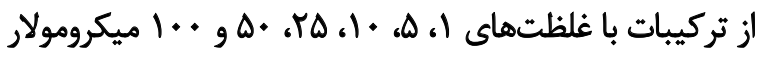

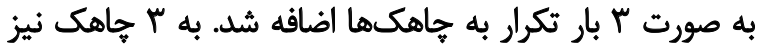

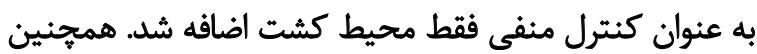

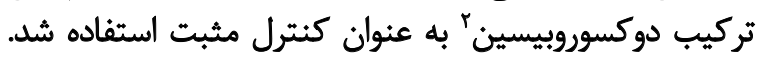

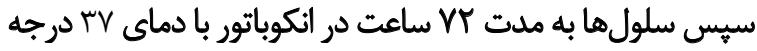

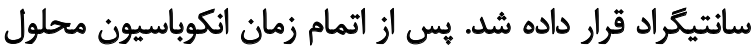

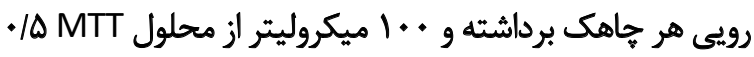

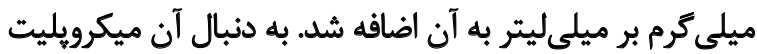

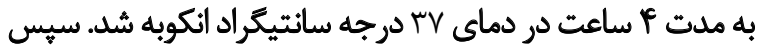

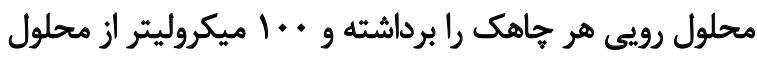

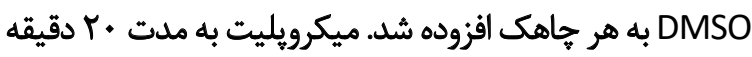

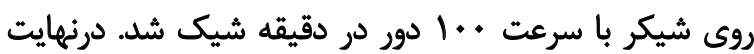

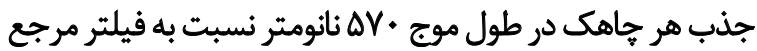
r بانومتر ثبت شد.

ميزان جذب نورى بلدست آمده بر اساس فرمول شماره 1

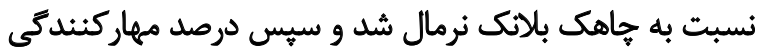

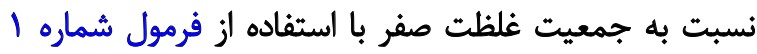

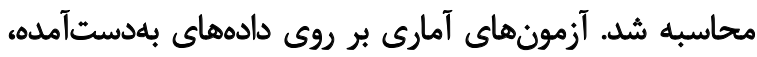

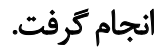

$$
\begin{aligned}
& O D_{\text {Treated }}=O D_{\text {Test }}-O D_{\text {Blank }} \\
& \text { × درصد جمعيت سلولى } 100=\left(O D_{\text {Treated }} / O D_{\text {Concentrationzero(untreated })}\right)
\end{aligned}
$$

بنابراين درصد مهاركنندكى طبق فرمول شماره r محاسبه
در DNA، مشتقات فتاليميد و نفتاليميد است. طراحى مواد ضد

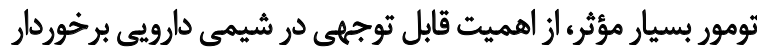

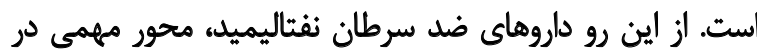

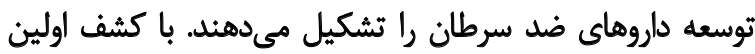

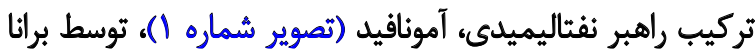

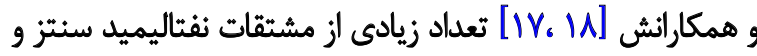

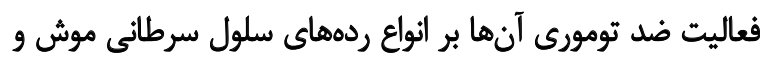

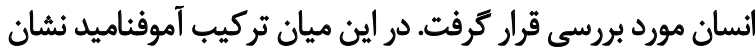

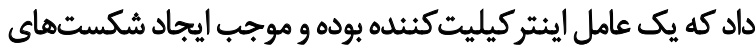

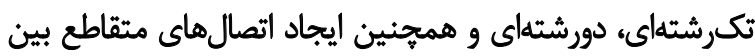

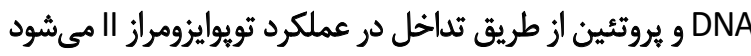

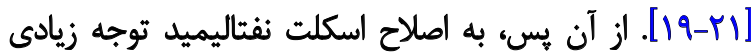

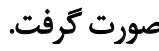
مشتقات اصلاحشده با سيستم جندحلقهاي آروماتيك تركيب

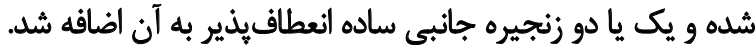

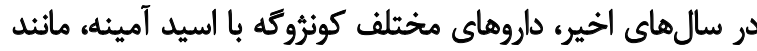

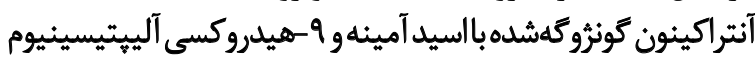
كزارش شد (تصوير شماره ))، كه نتايج اميدواركننده الى در اين اين راه

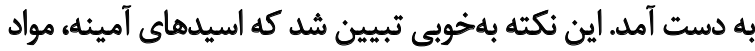

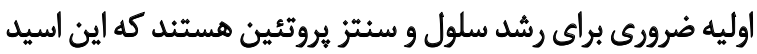

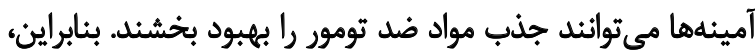

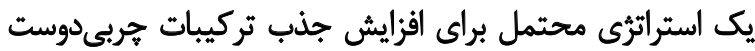

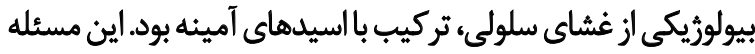

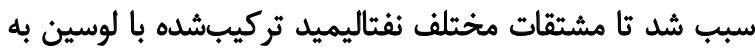

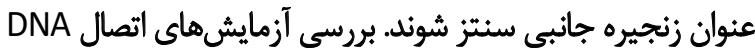

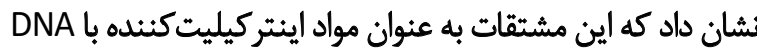

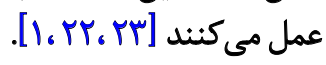
بنابراين با توجه به اهميت مشتقات نفتاليميد و فتاليميد و

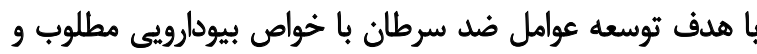

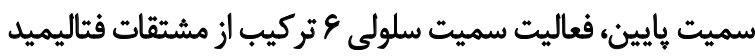

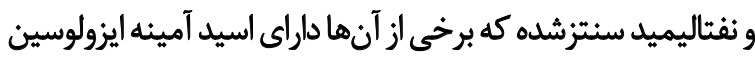

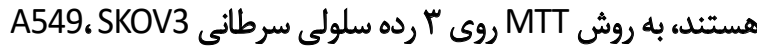

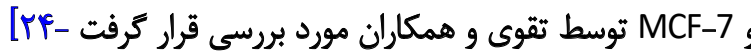

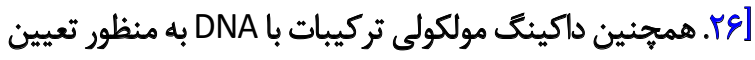

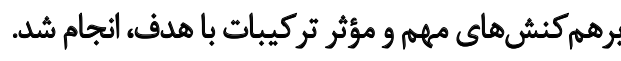

$$
\text { روش بروسى }
$$

در اين بخش ميزان فعاليت سيتوتوكسيك تركيبات با استفاده از

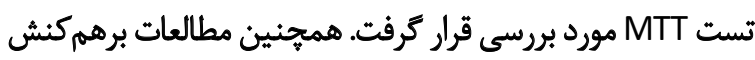

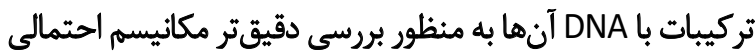

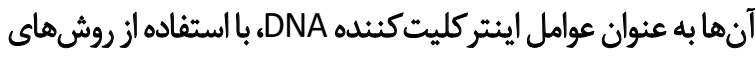

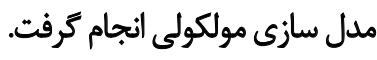




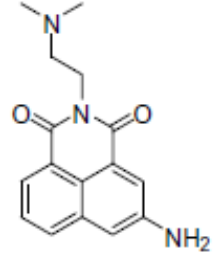

amonafide

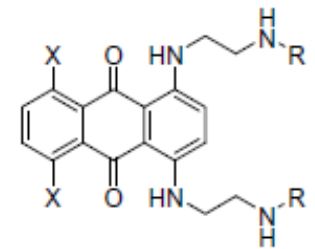

anthraquinone-amino acid conjugate

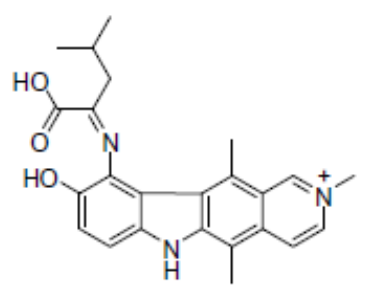

9-hydroxyellipticinium-Leu conjugate

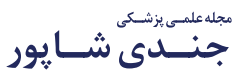

$$
\text { تصوير ا. ساختار آمونافيد، آنتراكينون كوتروكهشده با اسيد آميئه و 9-هيدروكسى الييتيسينيوم }
$$

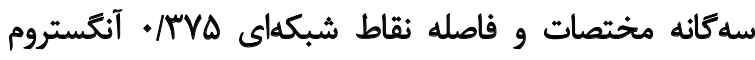

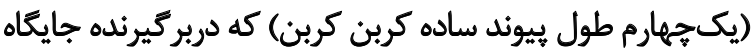

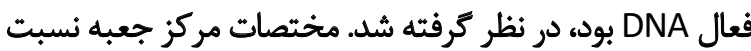

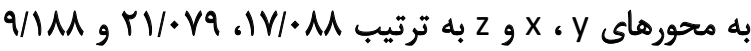

هن

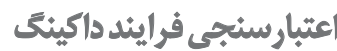

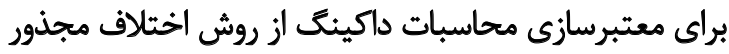

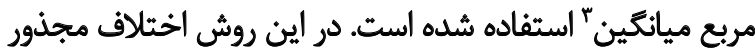

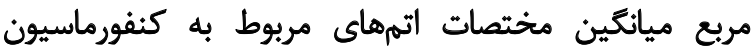

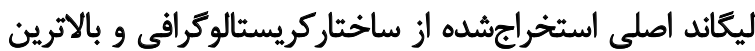

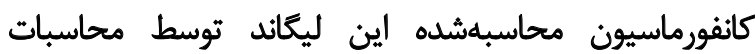

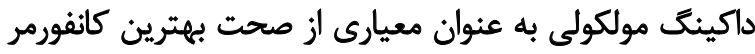
يُيشينيني

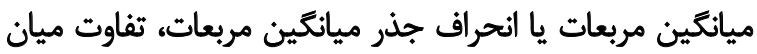

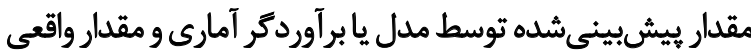

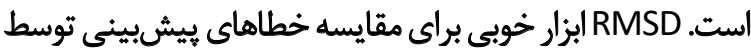

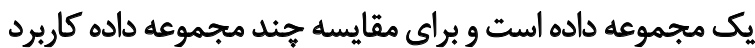

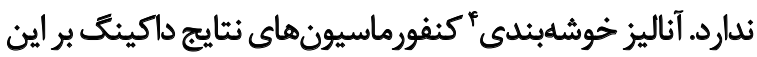

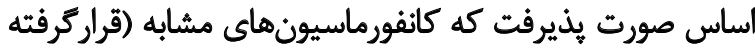

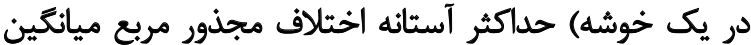
معادل r آنكستروم داشته باشند.

يافتهها

\section{ارويابى فعاليت سيثوتوكسيك تركيبات}

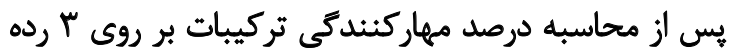

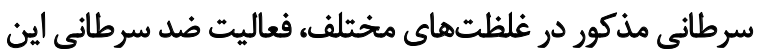

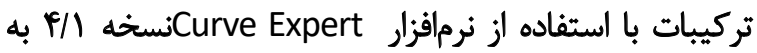
صورت IC محاسبه و در جدول شماره ا كزارش شدار

3. Root Mean Square Deviation

4. Cluster analysis

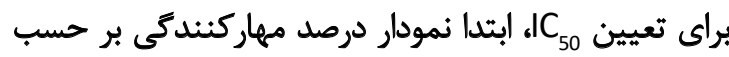

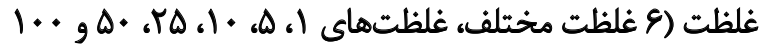

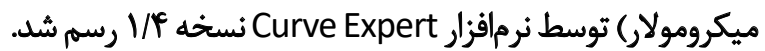

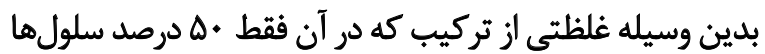

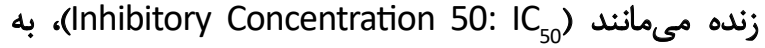

دست آمد.

\section{داكينََّ مولكولى}

مطالعات داكينگ مولكولى اين تركيبات توسط نرمافزار Autodock 1LU5 اكينگ، بهترين ساختار كريستالو

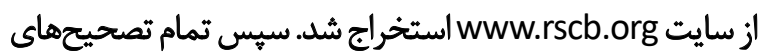

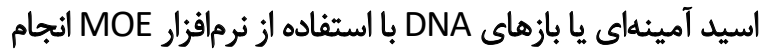

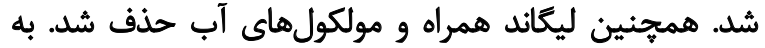

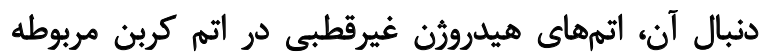

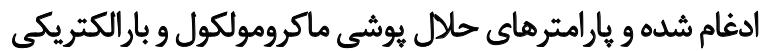

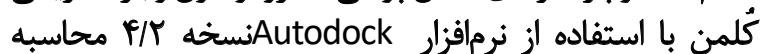

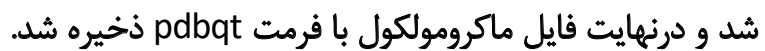

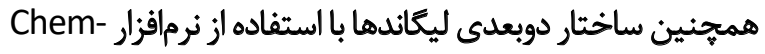

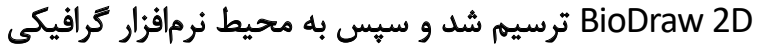
Hyperchem

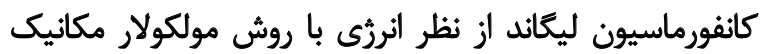

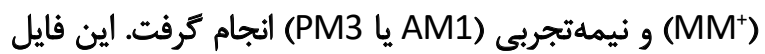

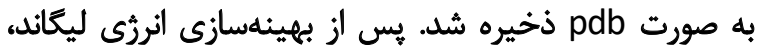

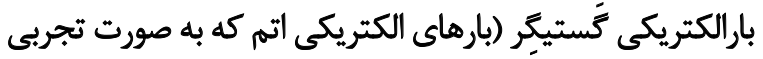

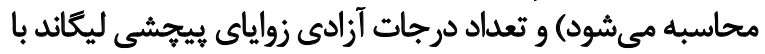

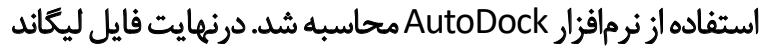

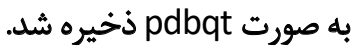

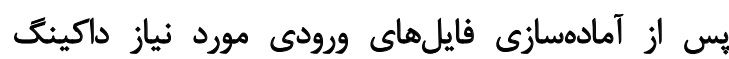

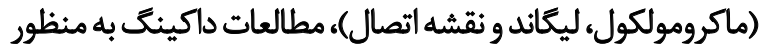

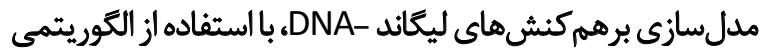

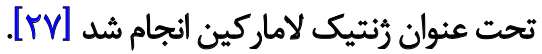
سيس بر اساس حجم مولكولى ليكائدهاى طراحى إشدهاه،

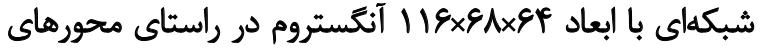




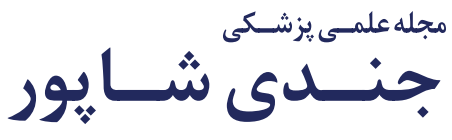

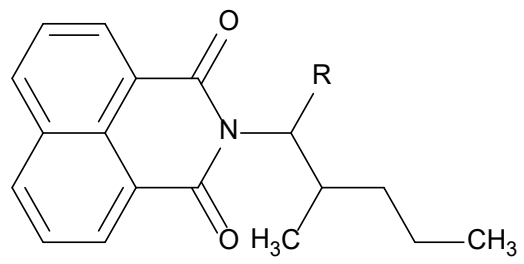

$\mathrm{C}_{1}-\mathrm{C}_{2}$<smiles>[R]C(Cc1ccccc1)N1C(=O)c2c(Br)c(Br)c(Br)c(Br)c2C1=O</smiles>

$\mathrm{C}_{3}-\mathrm{C}_{4}$<smiles>[R]C(Cc1ccccc1)N1C(=O)c2ccccc2C1=O</smiles>

$\mathrm{C}_{5}-\mathrm{C}_{6}$

جدول ا. نتايج ارزيابى زيستى به صورت

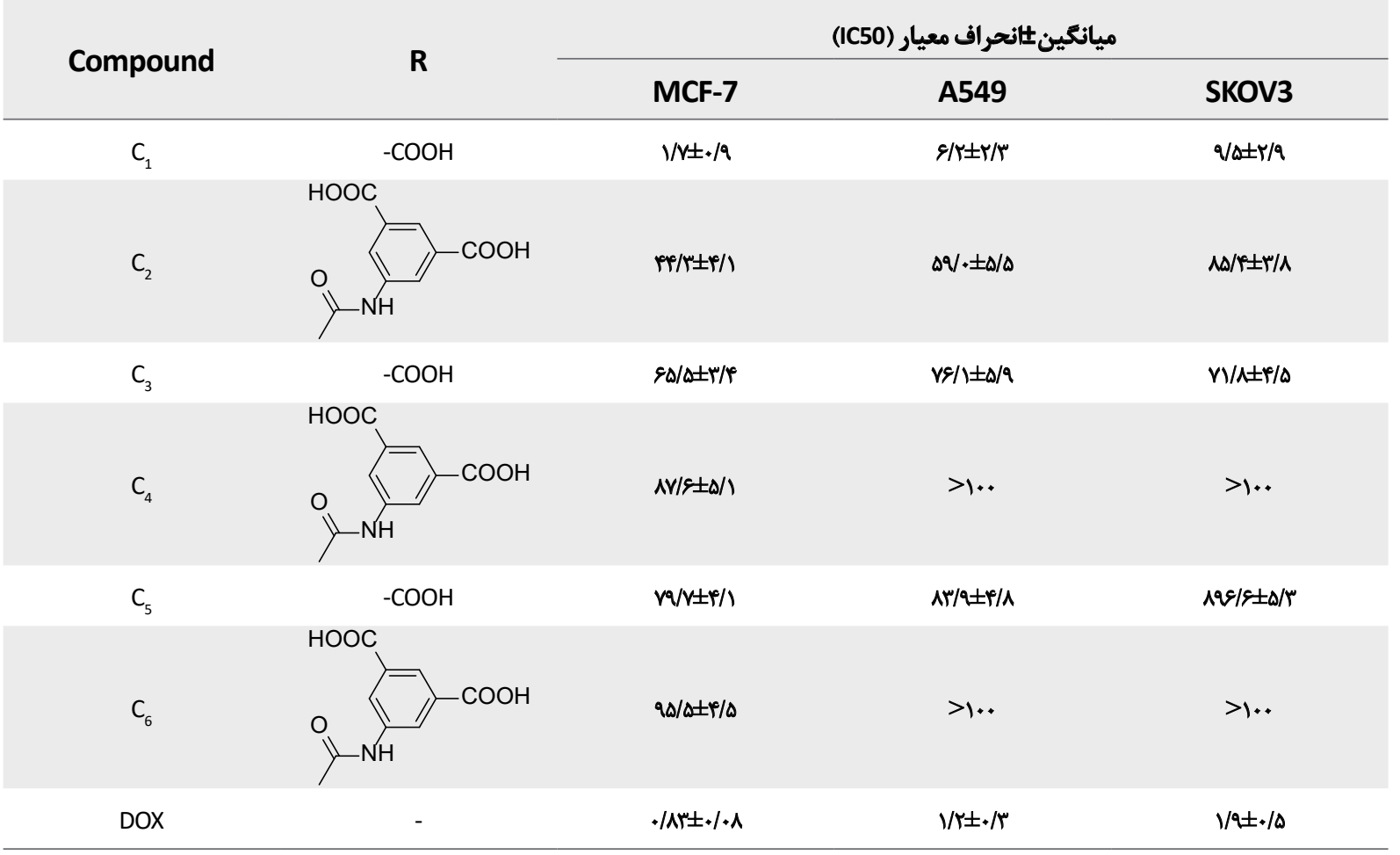

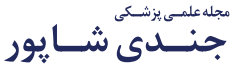

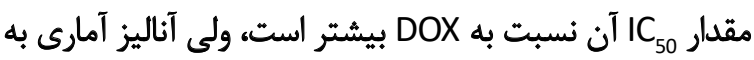

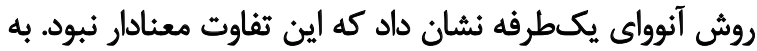

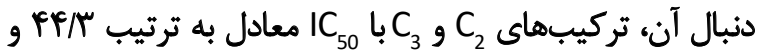

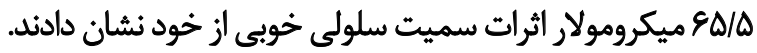

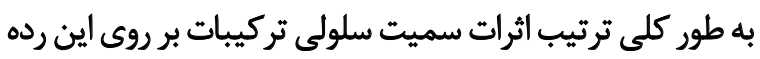
به شرح فرمول شماره بـ هستئد. r

MCF-7: $D O X>C_{1}>C_{3}>C_{5}>C_{3}>C_{4^{4}} C_{6}$

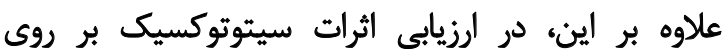

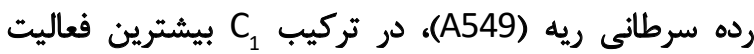

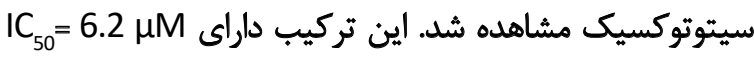

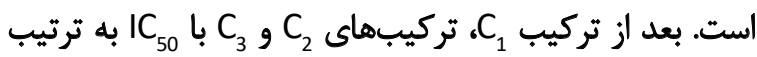

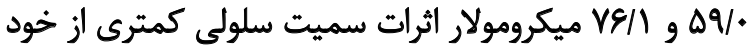
نشان دادند. ترتيب قدرت تركيبات بر روى اين رده به به شرح آرح

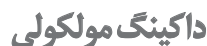

برهم كنش تركيبات با DNA (1LU5 توسط داكينخ مولكولى

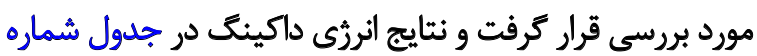

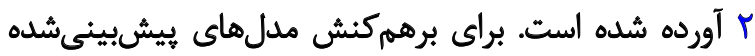

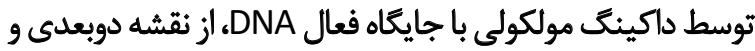

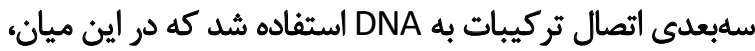

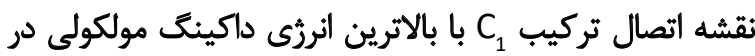
تصوير شماره rآورده شده است بالتي

$$
\text { بحث }
$$

با توجه به نتايج بهدست آمده در جدول شماره اه بيشترين

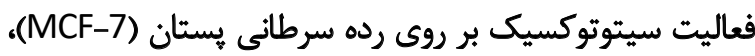

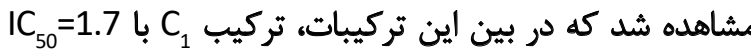

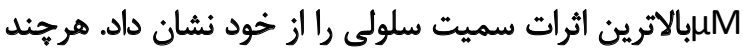




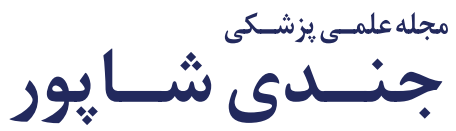

جدول ب. نتايج ائريى داكينّك مولكولى

\begin{tabular}{|c|c|}
\hline \multicolumn{2}{|c|}{ اتروى اتصال داكينى (كيلوكالرى برمول) } \\
\hline كيرنده (1LU5) & ل ايكاند \\
\hline$-V / N$ & $\mathrm{C} 1$ \\
\hline$-g / \Delta V$ & $\mathrm{C} 2$ \\
\hline$-g / \lambda_{*}$ & $\mathrm{C} 3$ \\
\hline זוI/ & $\mathrm{C} 4$ \\
\hline$-F / \Delta A$ & C5 \\
\hline g/Te & C6 \\
\hline
\end{tabular}

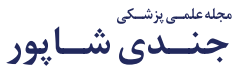

SKOV3: $\mathrm{DOX}>\mathrm{C}^{1}>\mathrm{C}^{3}>\mathrm{C}_{2}>\mathrm{C}_{5}>\mathrm{C}_{4^{\prime}}, \mathrm{C}_{6}$

به طور كلى تركيب C نسبت به بقيه تركيبات بر روى هر

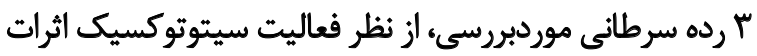

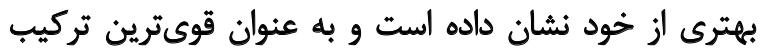
مجموعه عمل كرده است.

علاوه بر اين، مقايسه نتايج بهدستآمده در اين مطالعه بانتايج

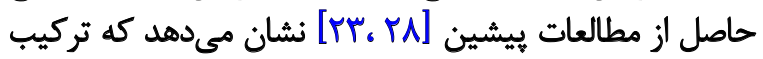

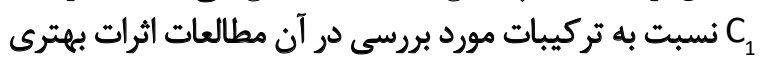

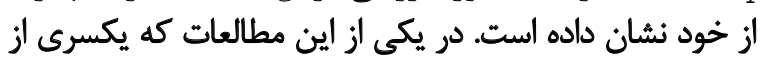

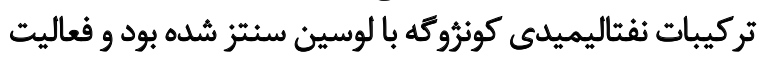

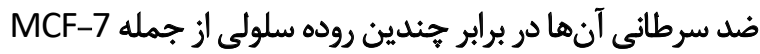

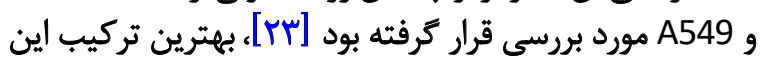

\begin{abstract}
A549: $\mathrm{DOX}>\mathrm{C}_{1}>\mathrm{C}_{2}>\mathrm{C}_{3}>\mathrm{C}_{5}>\mathrm{C}_{4}, \mathrm{C}_{6}$
همجنين در ارزيابي اثرات سيتوتوكسيك بر روى رده سرطاني

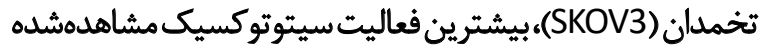

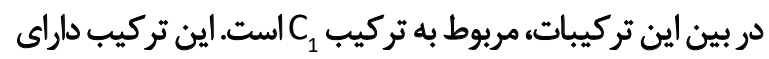

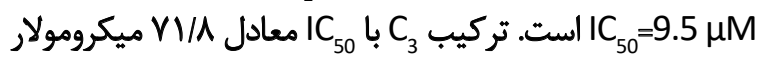
فعاليت سيتوتوكسيك ضعيفترى نسبت به تركيب C

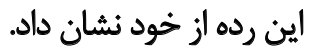
ترتيب قدرت تركيبات بر روى اين رده به شرح ذيل فرمول شماره ه است.
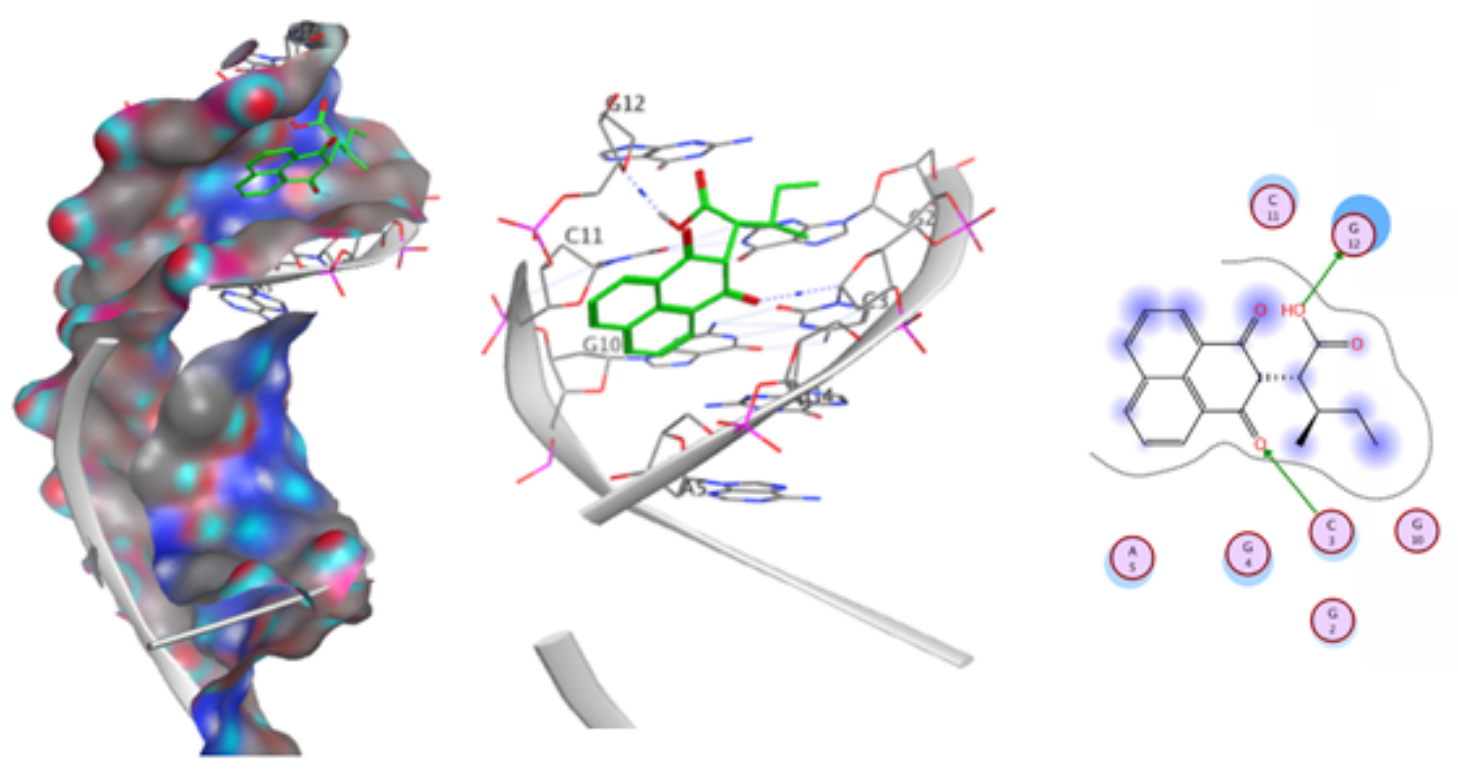

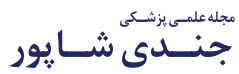

تصوير r. نمايش دوبعدى و سلبعدى نحوه برهم كنش تركيب C با جايگاه فعال 


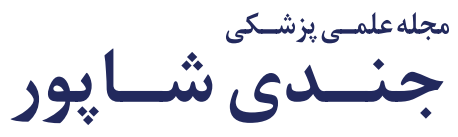

وجود اتصال به جايكاه فعال، برهمكنشهاى كليدى با بازها و يا

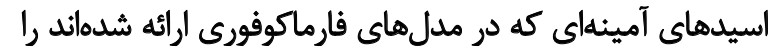

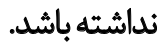

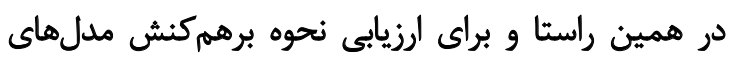

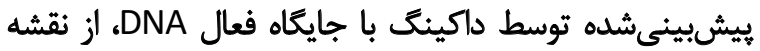

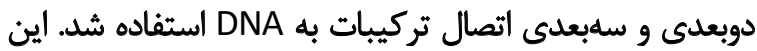

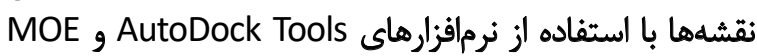

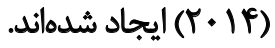

همان طور كه در تصوير شماره ب نشان داده شده است، تركيب

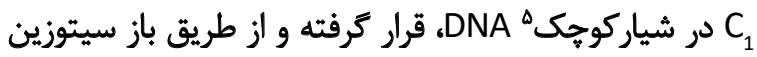

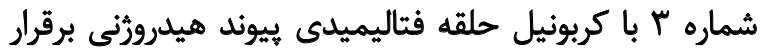

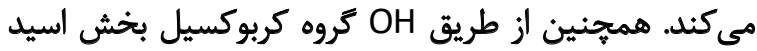

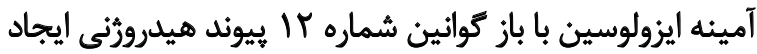

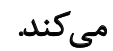

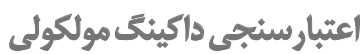

مطالعات داكينگ مولكولى در صورتى موفقيت آميز خواهد بود

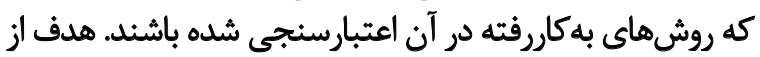

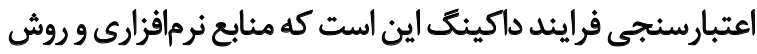

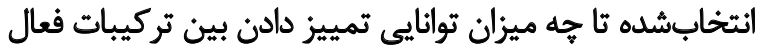

$$
\text { و غيرفعال را دارند. }
$$

روشهاى مختلفى براى اعتبارسنجى فرايند داكينگ و ورائ

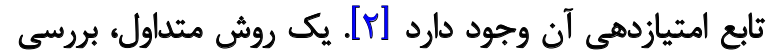

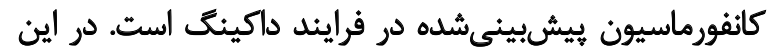

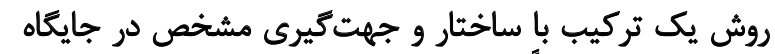

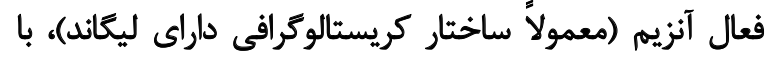

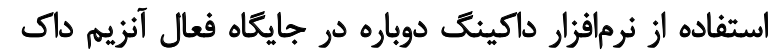

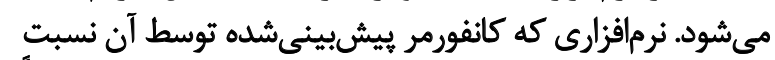

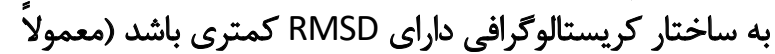

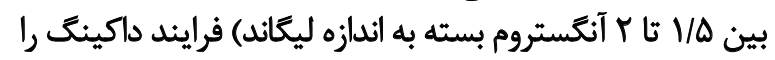

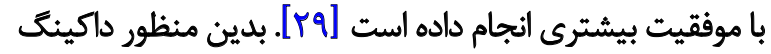

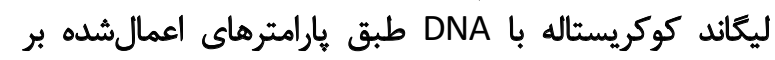

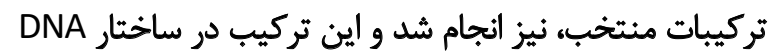

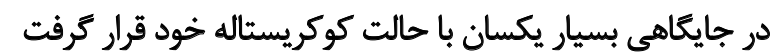

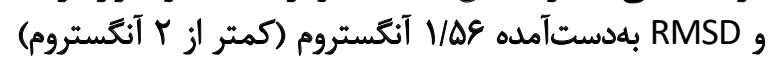
بوده است.

\section{نتيجهَيَيرى}

دراين مطالعه تجربى 9 تركيب فتاليمدى ونفتاليميدى بااثرات

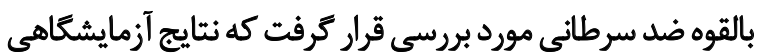

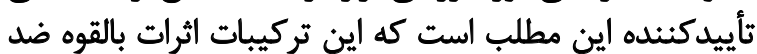

\section{Minor groove}

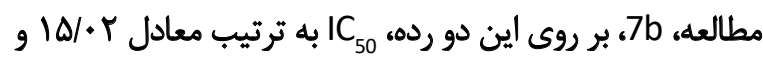

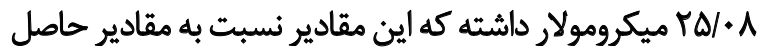

$$
\text { از تركيب C C Cيشتر است. }
$$

بروسى رابطله ساختار فاليث تر كياث

Cبرسى هاى اوليه نشان مى دهد كه تركيبات نفتاليميد (-)

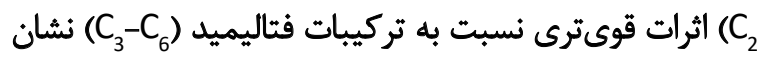

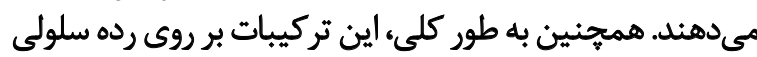

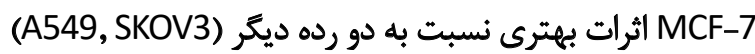

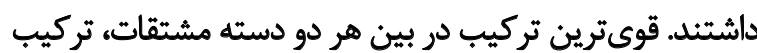

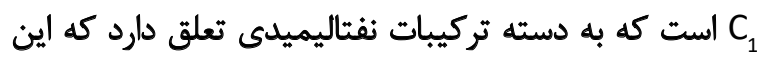

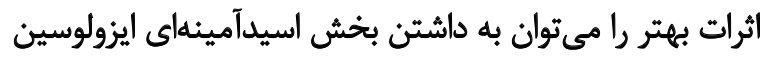

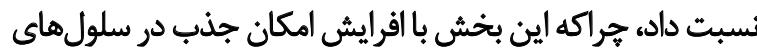

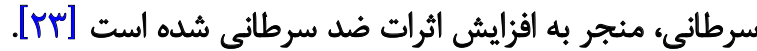

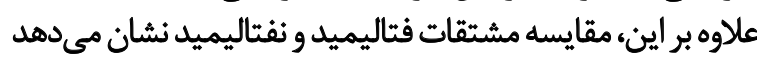

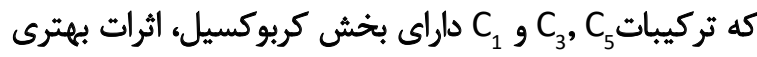

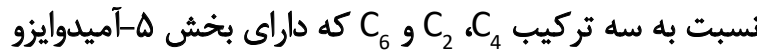

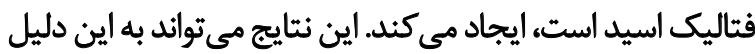

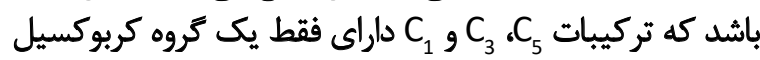

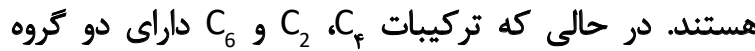

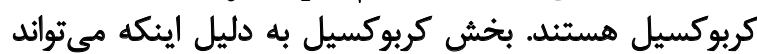
يونيده شود ميزان جذب تركيب را كاهش مى دهد.

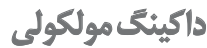

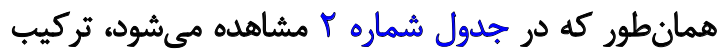

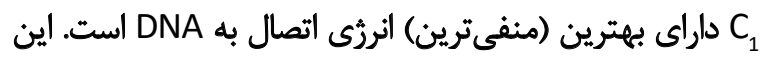

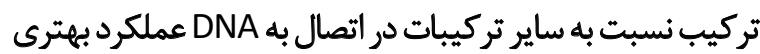

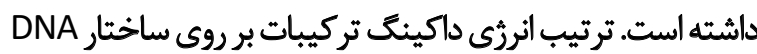
به شح فرمول شماره \& تراست.

DNA (1LU5): $\mathrm{C}_{1}>\mathrm{C}_{3}>\mathrm{C}_{5}>\mathrm{C}_{2}>\mathrm{C}_{6}>\mathrm{C}_{4}$

\section{بررسى الكوهاى بر همر كثش تر كيبات DNA}

بعد از مشخص شدن جايكاه هر ثركيب در رتبهبندى داكينك،

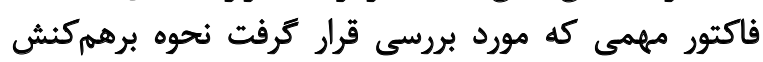

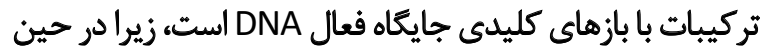

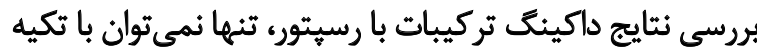

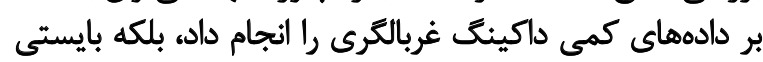

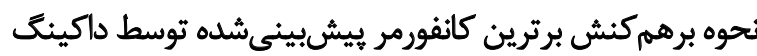

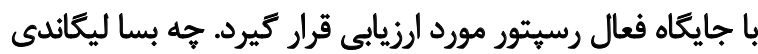

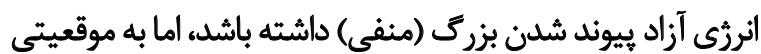

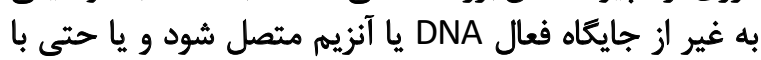


سرطانى از خود نشان مي دهند كه در ميان اين تركيبات، تركيب

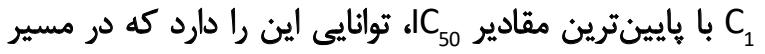

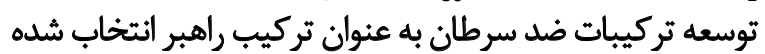
و مورد بررسى هاى بيشتر قرار تيرد. ملاحظات اخلاقى

\section{يبيروى أز اصول اخلاق يُوهش}

اين مطالعه مورد تأييد كميته اخلاق دانشكاه علوم يزشكى استى

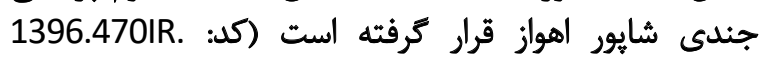
AJUMS.REC.

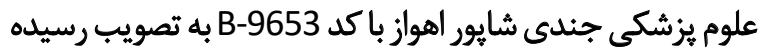

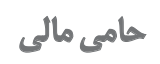

مقاله حاضر بخشى از رسالهى دكتراى حرفهاى داروسازى

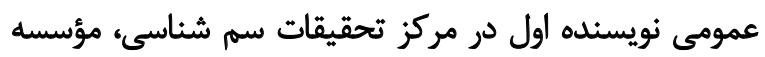

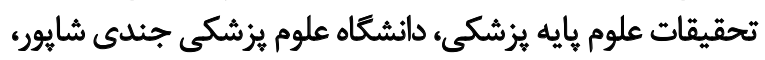

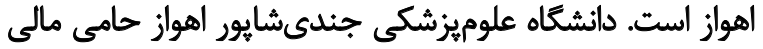

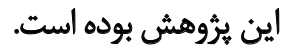
مشاركت نويسندكًان

ويراستارى، نهاييىسازى نوشته، تحقيق وبررسى:ايوب مجدمي؛

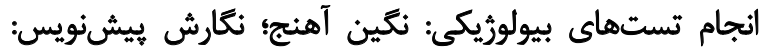

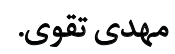

$$
\text { تعارض منافع }
$$

بنابر اظهار نويسندكان اين مقاله تعارض منافع ندارد.

$$
\text { تشكر وقدرداني }
$$

نويسندكان از دانشكاه علوميزشكى جندى شايور اهواز كه بودجه

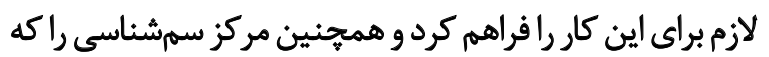
در اختيار محققان كذاشت، تشكر و قدردانى مى كنيند. 


\section{Refrences}

[1] Shankaraiah N, Kumar NP, Tokala R, Gayatri BS, Talla V, Santos LS. Synthesis of new 1,2,3-Triazolo-naphthalimide/ phthalimide conjugates via 'click' reaction: DNA intercalation and cytotoxic studies. J Braz Chem Soc. 2019; 30(3):454-61. [DOI:10.21577/0103-5053.20180111]

[2] Taheri B, Taghavi M, Zarei M, Chamkouri N, Mojaddami A. Imidazole and carbazole derivatives as potential anticancer agents: Molecular docking studies and cytotoxic activity evaluation. Bull Chem Soc Ethiop. 2020; 34(2):377-84. [DOI:10.4314/bcse.v34i2.14]

[3] Lima LM, Castro P, Machado AL, Fraga CAM, Lugnier C, de Moraes VLG, et al. Synthesis and anti-inflammatory activity of phthalimide derivatives, designed as new thalidomide analogues. Bioorg Med Chem. 2002; 10(9):3067-73. [DOI:10.1016/ S0968-0896(02)00152-9]

[4] Singh G, Saroa A, Girdhar Sh, Rani S, Sahoo S, ChoquesilloLazarte D. Synthesis, characterization, electronic absorption and antimicrobial studies of $\mathrm{N}$-(silatranylpropyl)phthalimide derived from phthalic anhydride. Inorganica Chim Acta. 2015; 427:232-9. [DOI:10.1016/j.ica.2015.01.011]

[5] Gong HH, Baathulaa K, Lv JS, Cai GX, Zhou CH. Synthesis and biological evaluation of Schiff base-linked imidazolyl naphthalimides as novel potential anti-MRSA agents. MedChemComm. 2016; 7(5):924-31. [DOI:10.1039/C5MD00574D]

[6] González MA, Clark J, Connelly M, Rivas F. Antimalarial activity of abietane ferruginol analogues possessing a phthalimide group. Bioorg Med Chem Lett. 2014; 24(22):5234-7. [DOI:10.1016/j.bmcl.2014.09.061] [PMID]

[7] Yang YJ, Zhao JH, Pan XD, Zhang PC. Synthesis and antiviral activity of phthiobuzone analogues. Chem Pharm Bull. 2010; 58(2):208-11. [DOI:10.1248/cpb.58.208] [PMID]

[8] Shinji Ch, Nakamura T, Maeda S, Yoshida M, Hashimoto Y, Miyachi $H$. Design and synthesis of phthalimide-type histone deacetylase inhibitors. Bioorg Med Chem Lett. 2005; 15(20):442731. [DOI:10.1016/j.bmcl.2005.07.048] [PMID]

[9] Si W, Zhang T, Zhang L, Mei X, Dong M, Zhang K, et al. Design, synthesis and bioactivity of novel phthalimide derivatives as acetylcholinesterase inhibitors. Bioorg Med Chem Lett. 2016; 26(9):2380-2. [DOI:10.1016/j.bmcl.2015.07.052] [PMID]

[10] Arif R, Nayab PS, Ansari IA, Shahid M, Irfan M, Alam Sh, et al. Synthesis, molecular docking and DNA binding studies of phthalimide-based copper(II) complex: In vitro antibacterial, hemolytic and antioxidant assessment. J Mol Struct. 2018; 1160:142-53. [DOI:10.1016/j.molstruc.2018.02.008]

[11] Kok SHL, Gambari R, Chui CH, Yuen MCW, Lin E, Wong RSM, et al. Synthesis and anti-cancer activity of benzothiazole containing phthalimide on human carcinoma cell lines. Bioorg Med Chem. 2008; 16(7):3626-31. [DOI:10.1016/j. bmc.2008.02.005] [PMID]

[12] Chan SH, Lam KH, Chui CH, Gambari R, Yuen MCW, Wong $\mathrm{RSM}$, et al. The preparation and in vitro antiproliferative activity of phthalimide based ketones on MDAMB-231 and SKHep-1 human carcinoma cell lines. Eur J Med Chem. 2009; 44(6):2736-40. [DOI:10.1016/j.ejmech.2008.10.024] [PMID]
[13] Kamal A, Bolla NR, Srikanth PS, Srivastava AK. Naphthalimide derivatives with therapeutic characteristics: A patent review. Expert Opin Ther Pat. 2013; 23(3):299-317. [DOI:10.1517/135 43776.2013.746313] [PMID]

[14] Rahmani-Nezhad S, Safavi M, Pordeli M, Kabudanian Ardestani S, Khosravani L, Pourshojaei Y, et al. Synthesis, in vitro cytotoxicity and apoptosis inducing study of 2-aryl-3-nitro-2H-chromene derivatives as potent anti-breast cancer agents. Eur J Med Chem. 2014; 86:562-9. [DOI:10.1016/j.ejmech.2014.09.017] [PMID]

[15] Pourshojaei Y, Abiri A, Eskandari R, Dourandish F, Eskandari $\mathrm{Kh}$, Asadipour A. Synthesis, biological evaluation, and computational studies of novel fused six-membered O-containing heterocycles as potential acetylcholinesterase inhibitors. Comput Biol Chem. 2019; 80:249-58. [DOI:10.1016/j.compbiolchem.2019.04.004] [PMID]

[16] Mirzaie V, Ansari M, Nematollahi-Mahani SN, Moballegh Nasery M, Karimi B, Eslaminejad T, et al. Nano-graphene oxide-supported APTES-Spermine, as gene delivery system, for transfection of pEGFP-p53 into breast cancer cell lines. Drug Des Devel Ther. 2020; 14:3087-97. [DOI:10.2147/DDDT. S251005] [PMID] [PMCID]

[17] Braña MF, Ramos A. Naphthalimides as anticancer agents: Synthesis and biological activity. Curr Med Chem Anticancer Agents. 2001; 1(3):237-55. [DOI:10.2174/1568011013354624] [PMID]

[18] Braña MF, Cacho M, Gradillas A, de Pascual-Teresa B, Ramos A. Intercalators as anticancer drugs. Curr Pharm Des. 2001; 7(17):1745-80. [DOI:10.2174/1381612013397113] [PMID]

[19] Hurley LH. DNA and its associated processes as targets for cancer therapy. Nat Rev Cancer. 2002; 2(3):188-200. [DOI:10.1038/nrc749] [PMID]

[20] Chen Zh, Liang X, Zhang H, Xie H, Liu J, Xu Y, et al. A new class of naphthalimide-based antitumor agents that inhibit topoisomerase II and induce lysosomal membrane permeabilization and apoptosis. J Med Chem. 2010; 53(6):2589-600. [DOI:10.1021/jm100025u] [PMID]

[21] Filosa R, Peduto A, Di Micco S, de Caprariis P, Festa M, Petrella $A$, et al. Molecular modelling studies, synthesis and biological activity of a series of novel bisnaphthalimides and their development as new DNA topoisomerase II inhibitors. Bioorg Med Chem. 2009; 17(1):13-24. [DOI:10.1016/j.bmc.2008.11.024] [PMID]

[22] Berridge MV, Herst PM, Tan AS. Tetrazolium dyes as tools in cell biology: New insights into their cellular reduction. Biotechnol Annu Rev. 2005; 11:127-52. [DOI:10.1016/S13872656(05)11004-7]

[23] Wu A, Xu Y, Qian X. Novel naphthalimide-amino acid conjugates with flexible leucine moiety as side chain: Design, synthesis and potential antitumor activity. Bioorg Med Chem. 2009; 17(2):592-9. [DOI:10.1016/j.bmc.2008.11.080] [PMID]

[24] Mallakpour Sh, Taghavi M. A facile, microwave-assisted synthesis of novel optically active polyamides derived from 5-(3-methyl-2-phthalimidylpentanoylamino)isophthalic acid and different diisocyanates. Eur Polym J. 2008; 44(1):87-97. [DOI:10.1016/j.eurpolymj.2007.10.027] 
[25] Mallakpour Sh, Taghavi M. Efficient and rapid synthesis of optically active polyamides in the presence of tetrabutylammonium bromide as ionic liquids under microwave irradiation. J Appl Polym Sci. 2008; 109(6):3603-12. [DOI:10.1002/ app.28263]

[26] Mallakpour Sh, Taghavi M. Molten tetrabutylammonium bromide as eco-friendly media for the synthesis of optically active and thermal stable polyamides under microwave irradiation. Polym J. 2008; 40(11):1049-59. [DOI:10.1295/polymj. PJ2008056]

[27] Khaledi M, Ziyaee Qychan Atiq H, Chamkouri N, Mojaddami A. Molecular docking and druggability studies of terpenoidderived metabolites from marine sponges as IL-17A inhibitors. Eurasian Chem Commun. 2019; 1(5):419-32. [DOI:10.33945/ SAMI/ECC.2019.5.2]

[28] de Oliveira Cardoso MV, Moreira DRM, Oliveira Filho GB, Cavalcanti SMT, Coelho LCD, Espíndola JWP, et al. Design, synthesis and structure-activity relationship of phthalimides endowed with dual antiproliferative and immunomodulatory activities. Eur J Med Chem. 2015; 96:491-503. [DOI:10.1016/j. ejmech.2015.04.041] [PMID]

[29] Fereidoonnezhad M, Tabaei SMH, Sakhteman AH, Seradj $H$, Faghih Z, Faghih Z, et al. Design, synthesis, molecular docking, biological evaluations and QSAR studies of novel dichloroacetate analogues as anticancer agent. J Mol Struct. 2020; 1221:128689. [DOI:10.1016/j.molstruc.2020.128689] 